\title{
Gaze-Dependent Visual Neurons in Area V3A of Monkey Prestriate Cortex
}

\author{
Claudio Galletti and Piero Paolo Battaglini ${ }^{a}$ \\ Cattedra di Fisiologia generale della Facoltá di Farmacia, Istituto di Fisiologia umana, Universitá di Bologna, 40127 \\ Bologna, Italy
}

\begin{abstract}
Extracellular recordings from single neurons of the prestriate area V3A were carried out in awake, behaving monkeys, to test the influence of the direction of gaze on cellular activity. The responsiveness to visual stimulation of about half of the studied neurons $(88 / 187)$ was influenced by the animal's direction of gaze: physically identical visual stimuli delivered to identical retinotopic positions (on the receptive field) evoked different responses, depending upon the direction of gaze. Control experiments discount the possibility that the observed phenomenon was due to changes in visual background or in depth, depending on the direction in which the animal was looking. The gaze effect modulated cell excitability with different strengths for different gaze directions. The majority of these neurons were more responsive when the animal looked contralaterally with respect to the hemisphere they were recorded from. Gaze-dependent neurons seem to be segregated in restricted cortical regions, within area V3A, without mixing with non-gaze-dependent cells of the same cortical area. The most reliable differences between V3A gaze-dependent neurons and the same type of cells previously described in area 7 a (Andersen and Mountcastle, 1983) concern the small receptive field size, the laterality of gaze effect, and the lack of straight-ahead facilitated or inhibited neurons in area V3A.
\end{abstract}

Since the present results show that V3A gaze-dependent neurons combine information about the position of the eye in the orbit with that of a restricted retinal locus (their receptive field), we suggest that they might directly encode spatial locations of the animal's field of view in a head frame of reference. These cells might be involved in the construction of an internal map of the visual environment in which the topographical position of the objects reflects their objective position in space instead of reflecting the retinotopic position of their images. Such an objective map of the visual world might allow the stability of visual perception despite eye movement.

Received Dec. 28, 1987; revised Aug. 10, 1988; accepted Aug. 17, 1988.

This work was supported by grants from the Ministero della Pubblica Istruziunte and CNR, Rome, Italy. We are grateful to V. B. Mountcastle for critical comments on an earlier version of the manuscript and to G. F. Poggio and S. Zeki for their cncouragement. We are also indebted to an anonymous referee of this journal for helpful comments that greatly improved the understanding of the paper. We thank A. Meoni for technical assistance during the experiments and Chemical Industries Bracco S.p.A. for having supplied the neurosurgical cement.

Correspondence should be addressed to C. Galletti, Istituto di Fisiologia umana, Piazza di Porta S. Donato 2, 40127 Bologna, Italy.

a Present address: Istituto di Fisiologia, Universitá di Trieste, via Fleming 22, 34127 Trieste, Italy.

Copyright (c) 1989 Society for Neuroscience $0270-6474 / 89 / 041112-14 \$ 02.00 / 0$
During visual exploration of the environment, we continuously redirect our gaze from one object to another in the field of view to bring images of interest onto the fovea. Each time the eyes move, the images of the field of view impinge upon different parts of the retina, but despite such changes in retinal location, we perceive a stable visual world.

According to MacKay (1973), the stability of visual perception despite eye movement should be due to the existence of an "objective" internal map of the visual environment that is not altered by eye movements. MacKay postulated that visual perception could be the result of 2 independent processes, one that allows the perception of an image and the other that evaluates the coordinates of the perceived image in connection with concurrent eye movements. This second process would correct the effect of retinal shifts of visual images due to changes in the direction of gaze, in order to maintain the stability of visual perception despite eye movements. These 2 processes, logether, might build up an internal representation of the visual environment where the topographical position of the objects depends on their objective position in space, instead of depending on the retinotopic position of their images.

To build up such an objective map, neurons are needed that receive at least 2 kinds of input: a visual one, coming from receptive field activation and signaling a well-defined retinotopic position, and an eye-position input signaling the changes in the direction of gaze and, therefore, different positions in space of the objects whose images fall on the same retinotopic position (receptive field).

Visual neurons whose response to receptive field stimulation is influenced by the eye position in the orbit have been described in thalamic intralaminar nuclei of the cat (Schlag et al., 1980) and in the posterior parietal cortex of macaque monkeys (Andersen and Mountcastle, 1983). All these gaze-sensitive visual neurons had large receptive fields, and for all of them the hypothesis has been advanced that they could encode visual space in a head frame of reference instead of in a retinotopic one. In particular, it has been shown that the visual responsiveness of parietal neurons is a function of 2 variables: the angle of gaze and the response profile of the receptive field, these neurons not being equally responsive to stimulation of different parts of the receptive field during steady fixation. These 2 variables, taken together, might allow posterior parietal neurons to encode visual spatial locations in head-centered coordinates (see Andersen et al., 1985; Zipser and Andersen, 1988).

We wanted to test whether cells in relatively early parts of the visual pathway (and with small receptive fields) were infiuenced by an eye-position signal. We present evidence that the responsiveness to visual stimulation of about half the neurons 
of area V3A (a prestriate area of the monkey visual cortex; see Zeki, 1978) is influenced by the direction of gaze. These neurons, having small and well-defined receptive fields, might directly encode objective locations in space and therefore contribute to the construction of an objective map of the field of view. Preliminary results have already been published in abstract form (Aicardi et al., 1987; Battaglini et al., 1987).

\section{Materials and Methods}

Extracellular recordings from single neurons of area V3A were made from 5 hemispheres of 3 juvenile monkeys (Macaca fascicularis; 2.7$3.8 \mathrm{~kg}$ ) performing visual fixation tasks. The behavioral and electrophysiological methods used are described in detail elsewhere (Galletti et al., 1984, 1988). Briefly, the animals sat in a primate chair, the head restrained by a metal holder device firmly attached to the skull by Refobacin Palacos R (Merck) neurosurgical cement. The chair was placed in a light-tight enclosure, and the animals binocularly viewed a tangent screen $80^{\circ} \times 80^{\circ}$ in extent, $57 \mathrm{~cm}$ in front of their eyes. By using a color detection task (Galletti et al., 1984), the animals were trained to maintain the fixation of a $0.2^{\circ}$ spot of light rear-projected on to the screen for 2-6 sec, without reacting to any other visual stimulus concurrently presented on the screen itself. Glass-coated Elgiloy microelectrodes (Suzuki and Azuma, 1976) were guided through the dura matcr into the visual cortex by a microdrive installed daily on a closed chamber permanently implanted on the skull. Eye movements were detected by the scleral search coil technique (Fuchs and Robinson, 1966), improved as suggested by Judge et al. (1980). During fixation, a light stimulus of variable shape, size, and color was first used to map the receptive field and then to test the neural response to the visual stimulation described below. The light intensity of the stimulus was usually about $1.0 \log$ unit above the level of a white, uniform background of $1-2 \mathrm{~cd} / \mathrm{m}^{2}$. Both fixation spot and light stimulus could be positioned anywhere on the screen by mirrors mounted on galvanometers and moved in any direction at speeds up to about $1000 \mathrm{deg} / \mathrm{sec}$. Collection, storage, and offline analysis of data, as well as control of behavioral procedures during both training and recording sessions, were accomplished with an Apple II computer, in a way described elsewhere (Battaglini ct al., 1984; Galletti et al., 1984). The sample rate for action potentials was $0.5 \mathrm{msec}$ while that for eye positions was $5 \mathrm{msec}$. The computer automatically discarded trials where the animals performed inaccurate behavioral operations, while, if an animal's fixation was inadequate, the trial was discarded on- or off-line by the operator.

In order to test whether the responsiveness of V3A neurons to visual stimulation was influenced by the direction of gaze, that is, by the position of the fixation spot on the screen (the animal's head being restrained), a standard pattern of visual stimulation was carried out. At first, the monkey looked at the fixation spot projected on the screen in the animal's straight-ahead direction (Fig. 1, position E), while the cell's response to a stationary or moving stimulus (depending on cell preference) was tested several times (7-14 trials). Then, the fixation spot was horizontally and/or vertically displaced $20^{\circ}$ from its original position, to reach one of the other 8 positions shown by crosses in Figure 1. Accordingly, the light stimulus was displaced on the screen so as to activate the receptive field in the new position; the receptive field was mapped again and a second block of trials started, repeating the previous visual stimulation at a different location on the screen.

A total screen surface of $40^{\circ} \times 40^{\circ}$ was tested in this way in a random sequence of 9 different screen locations. The sequence was randomly changed from cell to cell. To better define the spatial distribution of gaze effect on cell responsiveness, several neurons were tested at more than the 9 standard positions, either within the standard $40^{\circ} \times 40^{\circ}$ screen area or even outside it, to the borders of the screen $\left(80^{\circ} \times 80^{\circ}\right)$. In standard stimulation, however, we decided to test gaze deviations not greater than $20^{\circ}$ from the straight-ahead position since, in animals with the head unrestrained, gaze shifts less than $20^{\circ}$ are accomplished almost exclusively with the eyes, the head being moved very little (Tomlison and Bahra, 1986).

Particular attention was paid to possible variations of spike amplitude and/or excitability of the cell under study. To check this, one or more blocks of stimulation were routinely repeated along the random sequence of different screen locations. If cell responsiveness in a certain screen position was significantly different from that previously tested

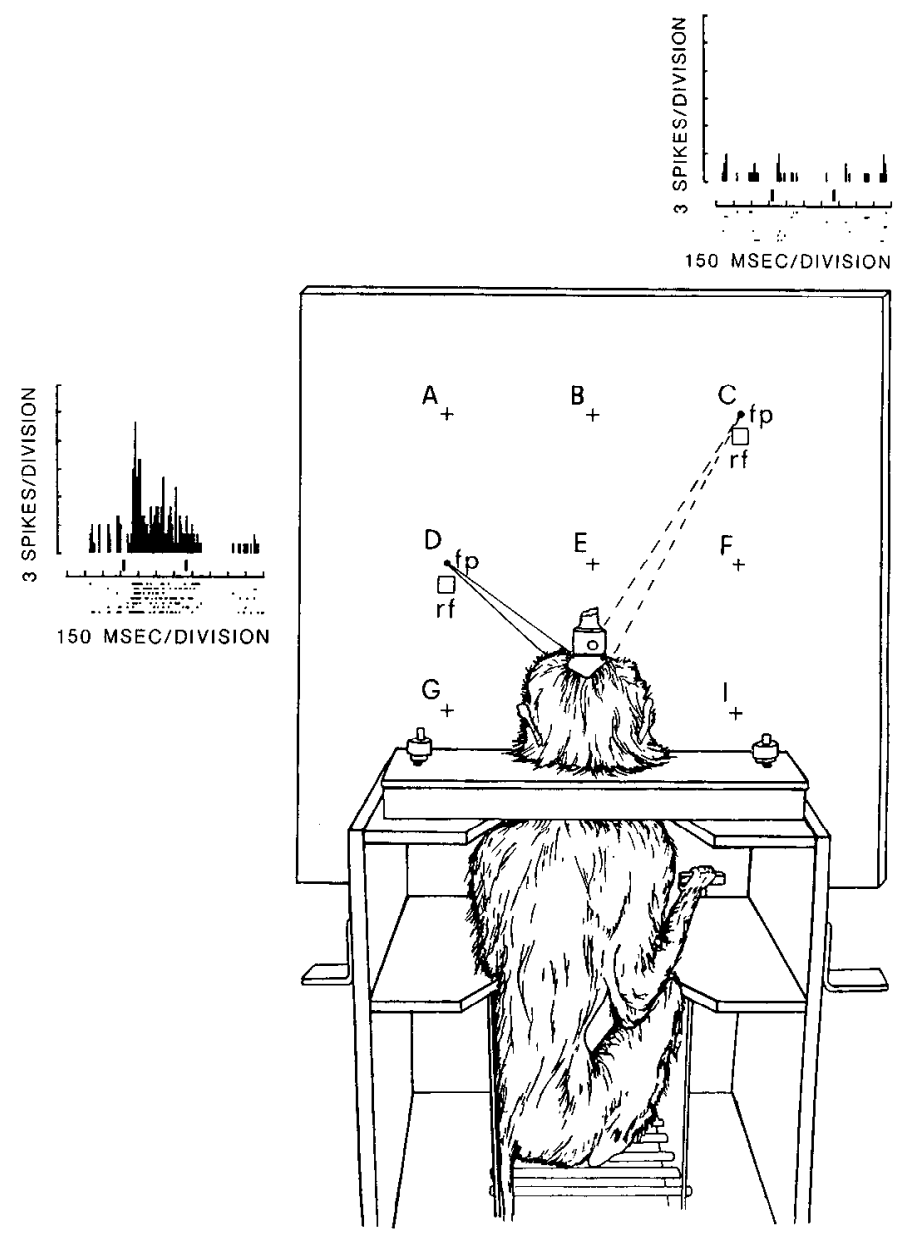

Figure 1. Experimental arrangement and effect of the direction of gazc upon the responses to visual stimulation of a V3A neuron. The animal faced an $80^{\circ} \times 80^{\circ}$ tangent screen with the head fixed (only a small part of the holder device has been shown) and gazed at a small spot of light $(f p$, fixation point) while the receptive field $(r f)$ of the cell under study was activated for $500 \mathrm{msec}$ by a correctly oriented stationary test stimulus of about the same size as the receptive field. Crosses from $A$ to $I$ show the 9 standard screen positions of the fixation point routinely used to test the gaze effect on each studied neuron. The visual response to receptive field activation during steady fixation at 2 of these positions $(C, D)$ are shown as peristimulus time histograms (PSTHs) and replicas of impulse sequences. Seven trials for each position were carried out. Landmarks under each PSTH indicate light-on and light-off, respectively.

in the same position, the sequence was interrupted and all data up to the previous control were discarded.

During the last recording sessions, electrolytic lesions $(20 \mu \mathrm{A} \times 30$ sec) were made at known coordinates within the chamber and at a known depth in the brain. Then the animals were deeply anesthetized, pins were inserted at known coordinates into the brain using the same microdrive that guided the microelectrode during recording sessions, and the animals were killed with an overdose of pentobarbital. Brains were removed, sectioned in a parasagittal plane, and stained with cresyl violet. Unmarked penetrations were reconstructed on the basis of their positions with respect to electrolytic lesions and pin tracks. More detailed information about surgery, histological technique, and reconstruction of microelectrode penetrations has been reportcd in a previous paper (Galletti et al., 1984).

\section{Results}

\section{Site of recording}

Reconstruction of microelectrode penetrations showed that neurons whose data are reported here were located in the annectant 
Figure 2. Reconstruction of 3 typical microelectrode penetrations. Bottom left, Camera lucida drawing of part of a sagittal section of the left hemisphere, taken at the level indicated in the outline of the hemisphere given at bottom right. In both drawings, anterior is left and posterior is right. Long bars on the section represent 3 penetrations reconstructed on a small number of successive sections and reported on the same drawing. Electrolytic lesions (open circles along a penetration track) allowed direct reconstruction of the penetration marked $b$. Penetrations $a$ and $c$ were indirectly reconstructed on the basis of the coordinates of pin tracks (not present in this section; see text) and penetration coordinatcs. Filled circles along penetration tracks indicate the recording sites of the cells whose receptive fields are shown in the 3 insets above the section. Note that outlines of receptive fields of V3A neurons are drawn bolder than those of $\mathrm{V} 3$ neurons. $V 1$, $V 2, V 3, V 3 A=$ cortical visual areas (dotted lines mark the limits of each); $A G=$ annectant gyrus; $C F=$ calcarine fissure; $L S=$ lunate sulcus.
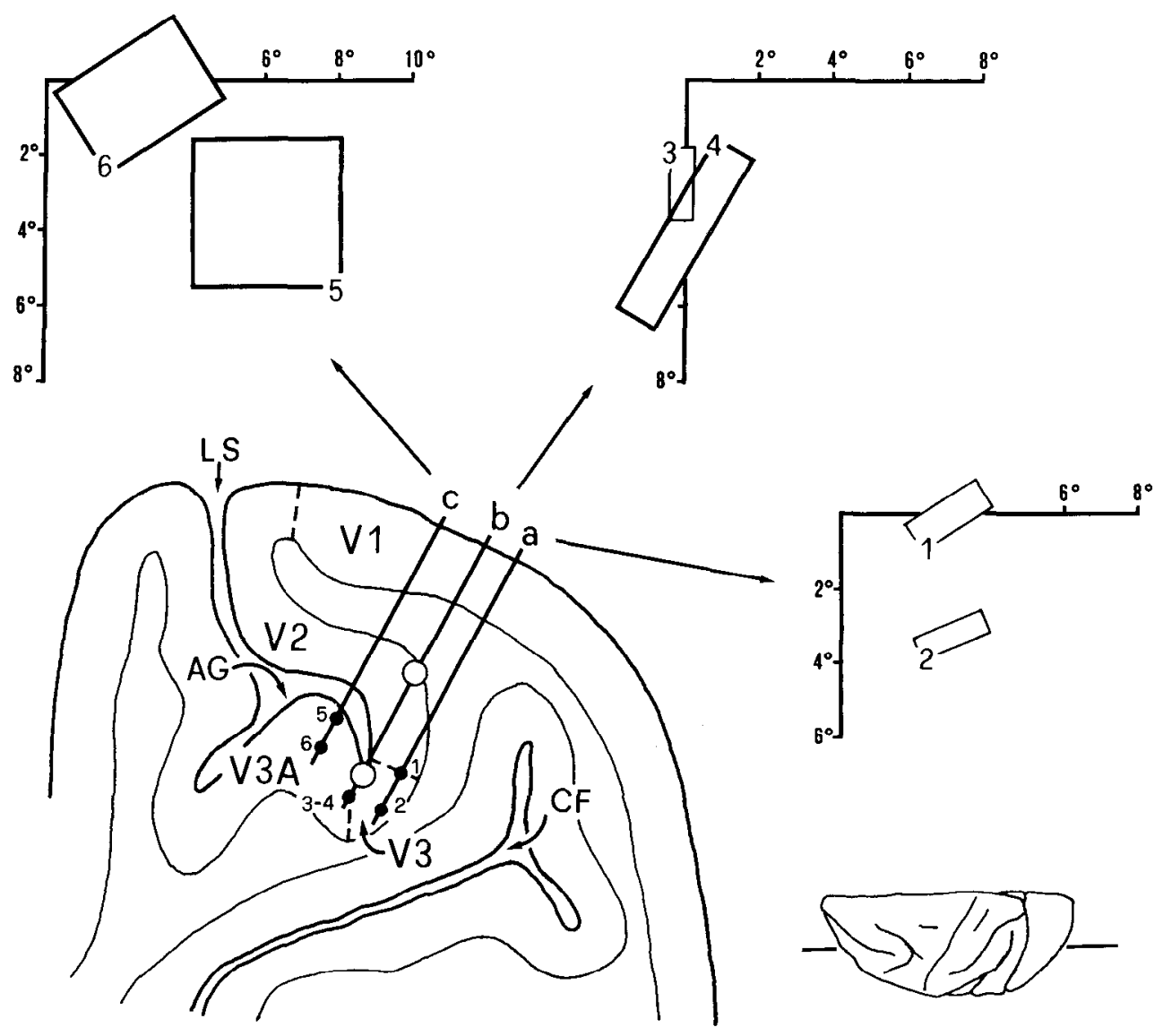

gyrus, which is buricd within the lunate sulcus and occupies part of its floor. According to Zeki and Sandeman (1976) and Van Essen and Zeki (1978), the visual field is partially represented at least twice in the annectant gyrus: in area V3, which mainly occupies the depth of the posterior bank of the lunate sulcus, and in area V3A, which occupies most of the annectant gyrus. The vertical meridian is represented between areas V3 and V3A, while the horizontal meridian is represented in the depth of the posterior bank of the lunate sulcus, between areas $\mathrm{V} 2$ and V3, and in the annectant gyrus, inside area V3A.

To decide whether each single neuron recorded during a microelectrode penetration was in area V3 or V3A, many neighboring penetrations had to be taken into account, looking at the shifts of receptive field locations according to depth readings and penetration coordinates. Figure 2 shows 3 penetrations carried out at different anteroposterior coordinates. The penetration marked " $a$ " passed through area V1, the underlying white matter, and again entered the gray matter at the depth of the posterior bank of the lunate sulcus. Moving down the lunate, receptive fields first approached the horizontal meridian (not shown in the figure), then moved away from it toward the vertical one (neurons 1-2). This suggested that recording was from area V3. Penetration " $b$," anterior to "a," passed through a first gray matter (area V1), a second one (area V2), and finally crossed the floor of the lunate sulcus into the annectant gyrus. Here, receptive fields were on the vertical meridian (neurons 3-4), hence at the border between areas V3 and V3A. We observed that, at the same eccentricity, receptive fields of V3A neurons were larger than those of V3 (see Fig. 3, left) and that the scatter of V3A neuron receptive fields along a single penetration was grcater than that observed in area V3. This allowed us to recognize from which of the 2 areas we were recording when receptive fields were close to the vertical meridian. The most anterior penetration " $c$ " in Figure 2 unambiguously reached, in depth, area $\mathrm{V} 3 \mathrm{~A}$, and, accordingly, receptive fields were large, showed great scatter and moved toward the horizontal meridian (neurons 5-6).

All the above reported considerations were laken into account to decide to which area a neuron belonged. In spite of this, it might be possible that our sample of V3A neurons is contaminated by a low percentage of $\mathrm{V} 3$ neurons whose receptive fields were located on the vertical meridian, that is, at the border with area V3A. As shown in the right part of Figure 3, the V3A neurons described in this paper had receptive fields located in the inferior quadrants of the visual field and their centers were within $15^{\circ}$ from the fixation point.

\section{Gaze-dependent neurons}

Out of a total of 207 cells located in area V3A, 187 were tested in at least 4 different screen positions. For the remaining 20 cells, either the angle of gaze studies were not done or fewer than 4 screen positions were tested, so they did not enter the neuronal population considered for the analysis. About $53 \%$ of V3A neurons (99/187) showed no significant differences in the responses evoked by visual stimulation of their receptive field in different positions on the screen: the excitability of these neurons did not seem to be significantly affected by the direction of gaze. In contrast, the excitability of about $47 \%$ of V3A neurons $(88 / 187)$ was clearly influenced by the direction of gaze.

The phenomenon and pattern of standard stimulation are 

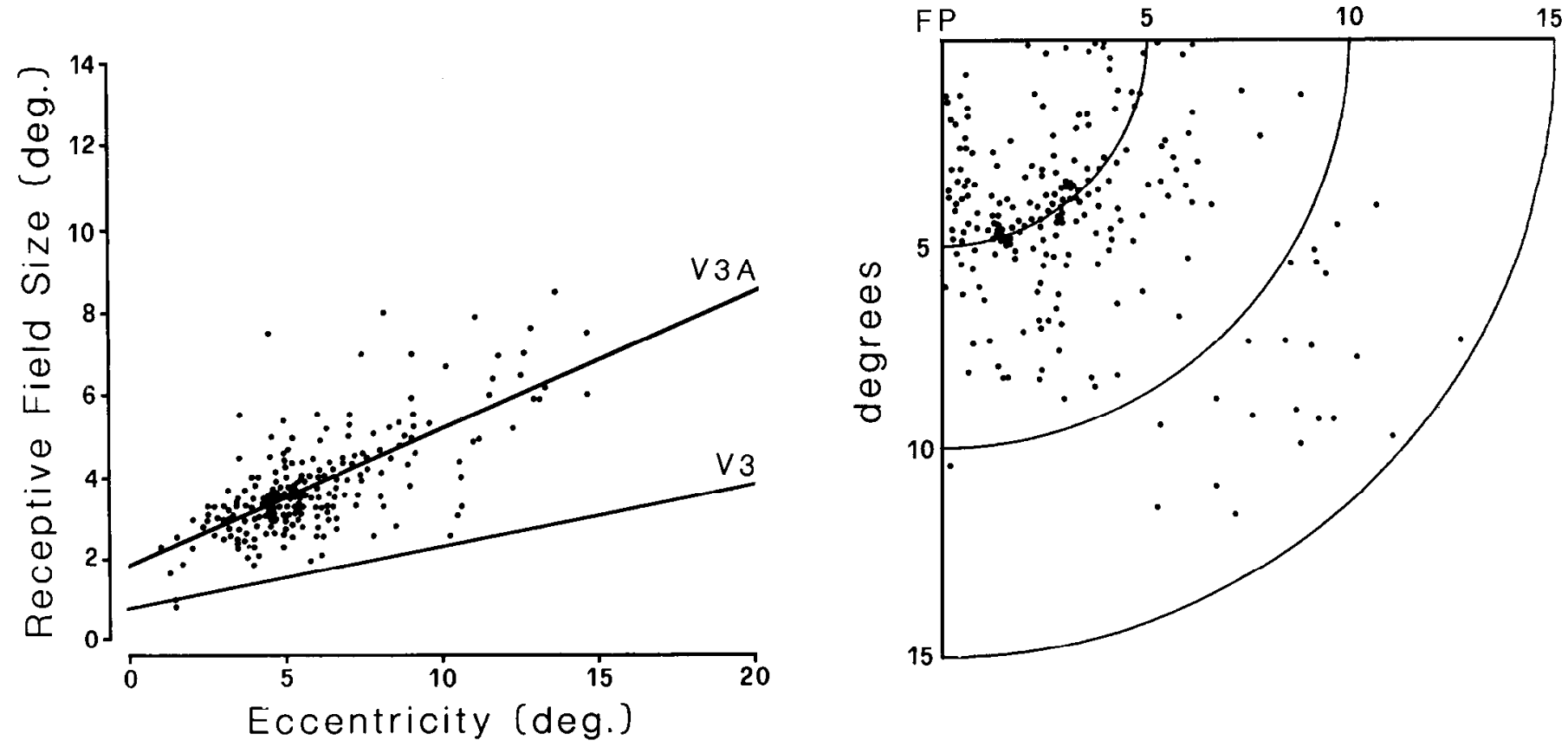

Figure 3. Receptive field size in areas V3 and V3A and extent of explored visual field in area V3A. Left, Distribution of receptive field size (square root of area) as a function of eccentricity for 207 neurons in area V3A and 44 in area V3. For arca V3, only the regression line has been shown for the sake of clarity. The least-squares regression functions for areas V3A and V3 were: RF size $(\mathrm{deg})=0.33 \times$ eccentricity $(\mathrm{deg})+1.78(r=$ $0.78)$ and RF size $(\mathrm{deg})=0.15 \times$ eccentricity $(\mathrm{deg})+0.91(r=0.70)$, respectively. Right, Distribution of receptive field centers of 207 neurons recorded from area V3A. Neurons recorded from both hemispheres are all reported in the right inferior quadrant of the visual field.

illustrated in Figure 1, in which the monkey and the tangent screen in front of it are shown as if seen from behind. As shown, the responses of only 2 of the 9 tested screen positions are shown for the sake of clarity. During fixation toward the left side of the screen (position D), a very good neuronal response was elicited by receptive field stimulation. On the contrary, the same physical stimulation of the receptive field did not evoke any response at all during fixation toward the right upper quadrant of the screen (position C). Although in this and some other cases certain directions of gaze completely abolished the cell's response to visual stimulation, changes in the direction of gaze did not usually produce all-or-none effects upon cell responsiveness. Rather, a gradual effect was usually observed over most of the animal's field of view.

Figure 4 shows the full field analysis of gaze effect carried out on the same neuron of Figure 1. This neuron was maximally responsive when the receptive field was stimulated while the animal looked toward the left side of the screen. There was a strong but gradual decrease of cell responsiveness when gaze was in other directions, particularly rightward and upward. When the animal looked toward the upper right corner of the screen ( $C$ in Figs. 1 and 4), we were unable to map any receptive field; the neuron was completely unresponsive to any kind of visual stimulation.

We have never observed ncurons whosc responsivencss was either maximal or minimal for fixation straight-ahead. All gazesensitive V3A neurons showed a planar distribution of responsiveness across the tested field of view, like that described in Figure 4. Figure 5 illustrates the distribution of responsiveness observed in 7 gaze-dependent neurons. The visual response at each tested screen position was calculated by subtracting the spontaneous activity of the cell (before visual stimulation) from the best $200 \mathrm{msec}$ firing rate obtained by the neuron during visual stimulation. The responses were then normalized and expressed as dark disks whose diameter was proportional to the visual response obtained by the receptive field stimulation in that particular position of the screen. The locations of the disks in each square (the screen) were those of the receptive field (instead of fixation point) in the field of view when the animal looked at different positions on the screen. Thus, the distribution of responsiveness for neurons with receptive field at different eccentricities may be compared in terms of the actual position in space. Unit 08.151 in Figure 5 is the same as that reported in Figures 1 and 4, in order to illustrate how the firing rate of discharge has been converted into dark disks of different sizes.

It is interesting to note that different patterns of spatial distribution were encountered in a very restricted region of cortex. Units 10.131 and 10.132, 10.119 and $10.120,10.123$ and 10.124 , for example, were recorded from 3 close-spaced microelectrode penetrations (about $1 \mathrm{~mm}$ apart). While in one of these penetrations 2 units, recorded $200 \mu \mathrm{m}$ apart, showed the same responsiveness distribution pattern (see units 10.131 and 10.132), in another penetration 2 units recorded at almost the same depth showed a different pattern (units 10.119 and 10.120); finally, in the third penetration, 2 units, recorded $400 \mu \mathrm{m}$ apart, showed just the opposite pattern of spatial distribution (units 10.123 and 10.124).

A more detailed analysis of the spatial distribution of neuronal responsiveness was performed for those neurons in which a greater number of screen locations were tested. Figure 6 shows 2 gaze-dependent neurons whose receptive field was stimulated while the animal was successively looking at 30 and 36 different 


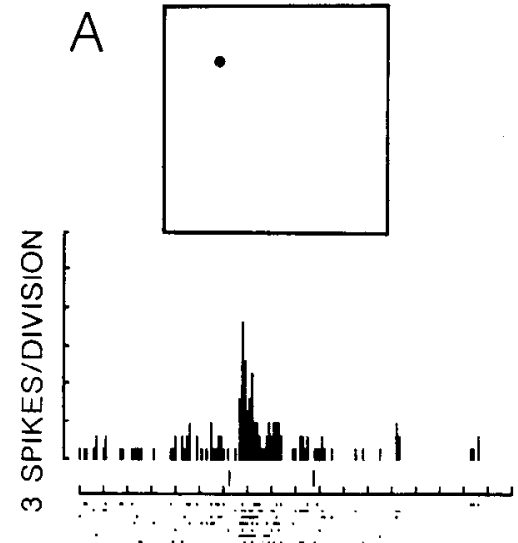

150 MSEC/DIVISION
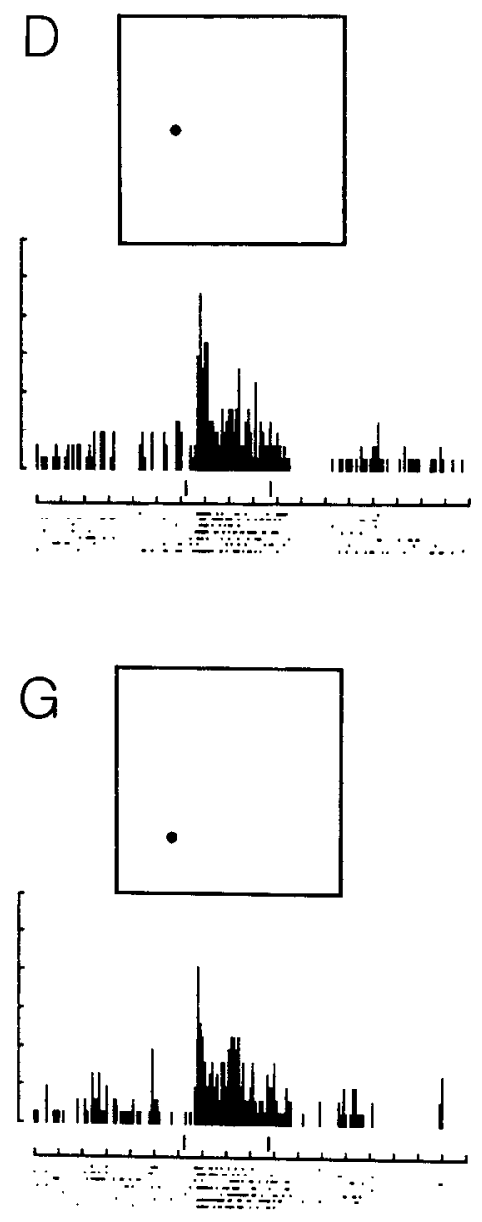
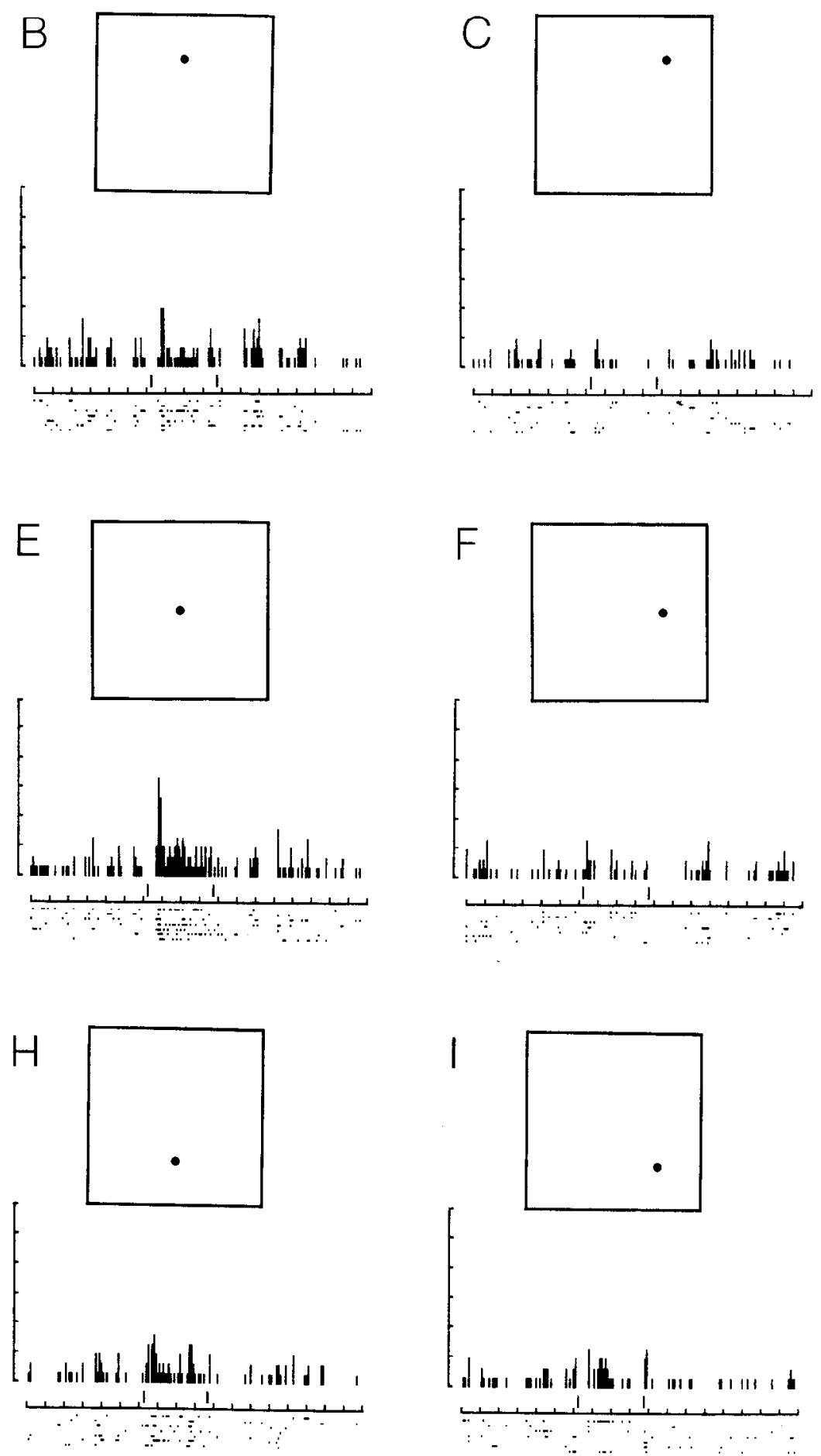

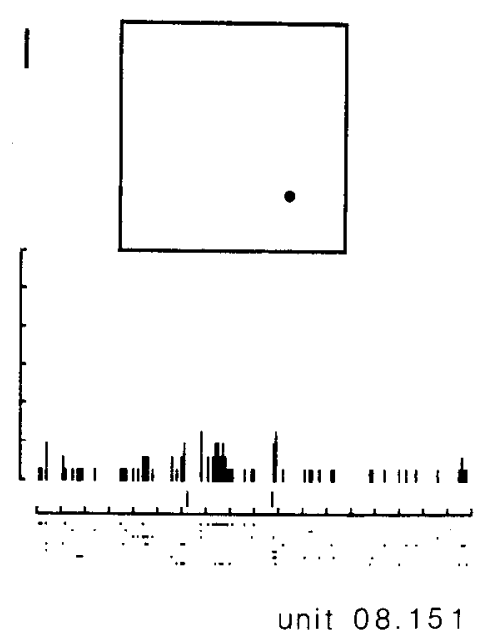

Figure 4. Influence of the direction of gaze upon the responsiveness of a V3A gaze-dependent neuron. The neuron was isolated in the annectant gyrus of the right hemisphere. Its receptive field $\left(2^{\circ} \times 2^{\circ}\right)$ was located at about $2^{\circ}$ from the fovea and overlapped the vertical meridian. $A$, The square represents the screen that was in front of the animal and the black spot inside it the position of the fixation point. Peristimulus time histogram (PSTH) and replicas of impulse sequences show the neuronal responses to 7 presentations of a 500 msec stationary visual stimulus of about the same size as the receptive field. Landmarks under the PSTH indicate light-on and light-off, respectively. $B-I$, Description as in $A$, the only difference being the position of the fixation point on the screen and, consequently, those of the receptive field and of the stimulus (not shown).

screen positions, respectively, instead of the standard nine. In Figure 6, dark disks have been replaced by isoresponsiveness lines. These lines link together different spatial locations whose stimulation evoked the same response when the receptive field was there in space, due to different directions of gaze. It can be observed that unit 08.150 shows a spotlike distribution of its responsiveness across the tested surface of $45^{\circ} \times 40^{\circ}$, while spatial distribution of unit 10.116 is much more smoothly distributed across a wider tested gaze field $\left(70^{\circ} \times 65^{\circ}\right)$. In any case, the firing rate of both cells is clearly dependent on 2 factors: the 


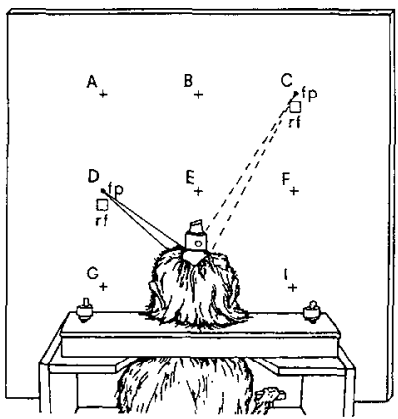

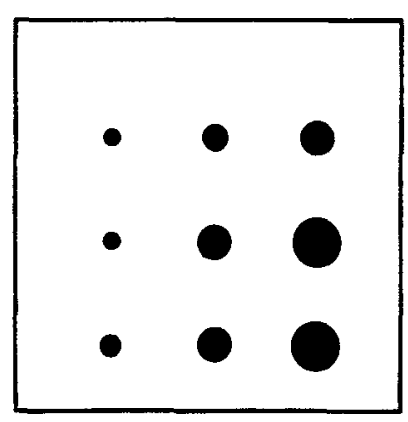

unit 10.131

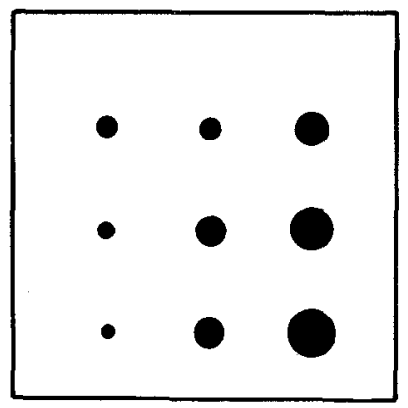

unit 10.132

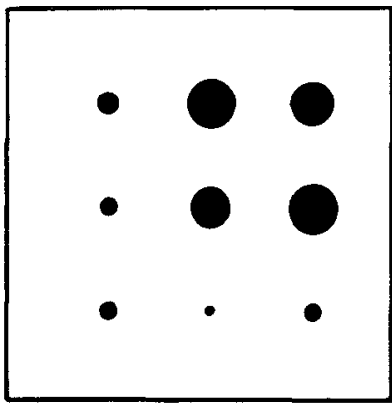

unit 10.119

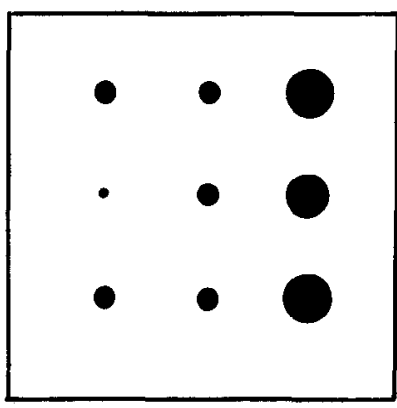

unit 10.120 unit 10.123
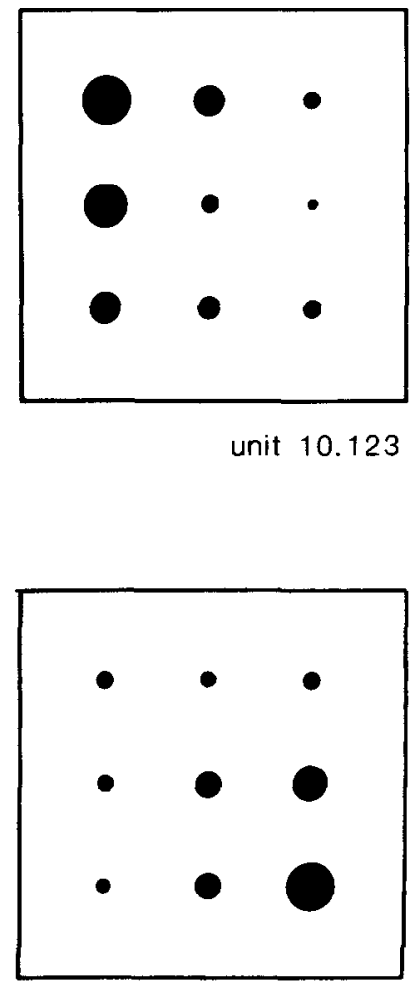

unit 10.124

Figure 5. Gaze-related distribution of responsiveness to visual stimulation of 7 V3A neurons. Top left, The experimental arrangement as described in Figure 1. In the remaining parts, the squares represent the screen in front of the animal and dark disks in each square indicate the locations of the receptive field on the screen when the animal looked at different screen positions. The diameters of dark disks are proportional to the visual responses evoked by receptive field stimulation in that particular screen position. Units 10.131 and 10.132 , units 10.119 and 10.120 and units 10.123 and 10.124 were recorded in 3 close-spaced penetrations.

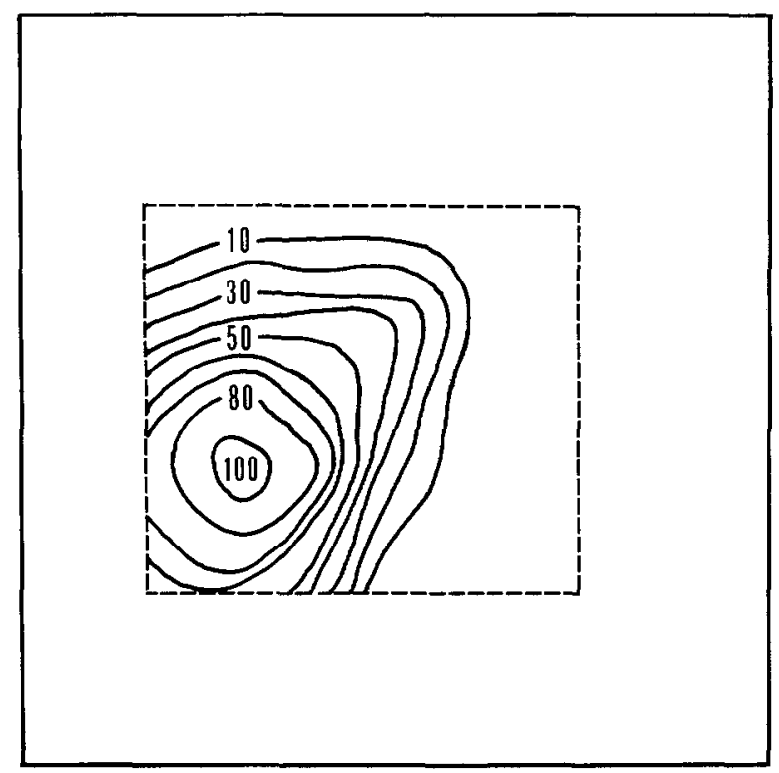

unit 08.150

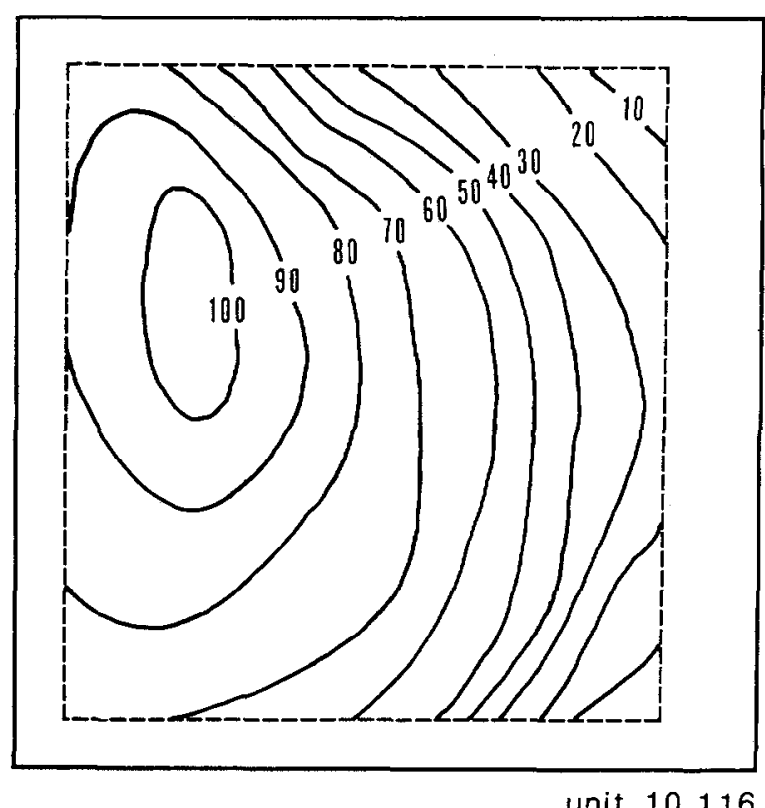

unit 10.116

Figure 6. Detailed analysis of the gaze-related distribution of responsiveness to visual stimulation of 2 V 3 A neurons. The large squares represent the screen in front of the animal. Dashed line inside each square outlines the tested gaze field, i.e., the part of the animal's field of view where the gaze effect was tested by displacing the fixation point. Thirty and thirty-six different screen positions have been tested for cells 08.150 and 10.116 , respectively. The visual responses to receptive field stimulation obtained at different fixation point positions are not shown. The lines inside the tested gaze fields are isoresponsiveness lines. They link different spatial locations whose stimulation cvoked the same response when the receptive field was there in space, due to different directions of gaze. Numbers on the lines quantify the visual responses as a percentage of their maximum value. 

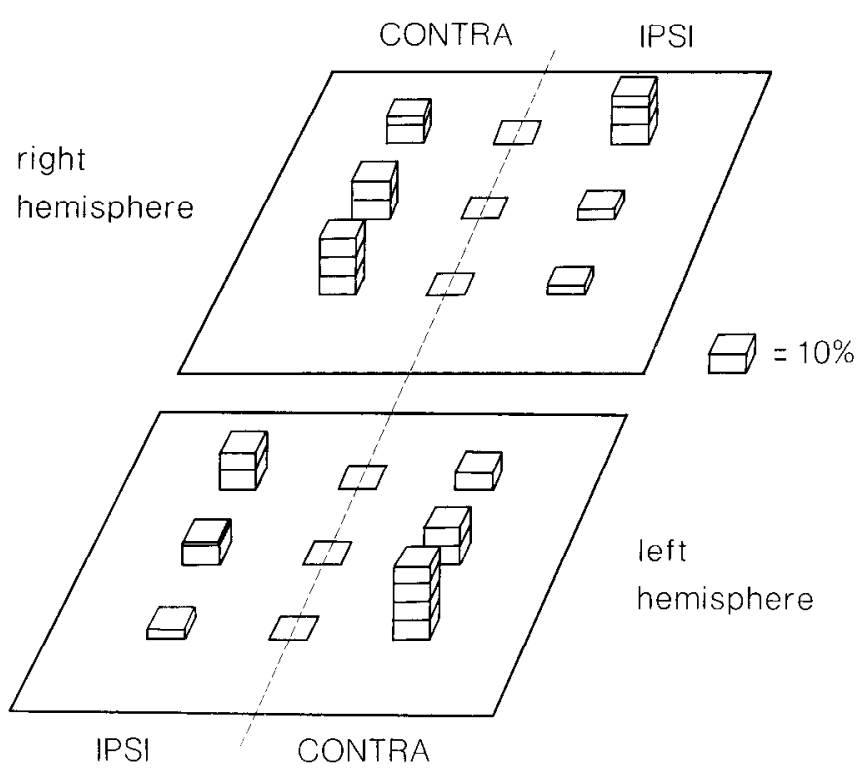

Figure 7. Plots of the fixation locations at which V3A gaze-dependent cells show the most vigorous response to visual stimulation. The results from the 2 hemispheres have been separately reported $(n=20$ for the right and $n=57$ for the left hemisphere). IPSI and CONTRA refer to regions of the field of view with respect to the hemisphere to which each neuron belonged. Dashed lines represent the vertical meridian when the animal looked at the center of the screen. Pillars on each of the 9 standard fixation locations represent the percentage of gaze-dependent cells best activated by receptive field stimulation when the animal looked in that particular direction. The squares reported in the straight-ahead position (the central one) and in both upward and downward directions in both hemispheres represent the "base" of a pillar, indicating that no cells were best responsive while the animal looked in those directions.

proper stimulation of a particular retinal locus (the receptive field) and the eye position in the orbit; this means that they are tuned for visual space locations in head-centered coordinates.

An attempt was made to quantify the change in responsiveness of the gaze-dependent neurons studied in at least 9 different screen positions $(n=77)$. For each neuron, we calculated all the "local" gradients of responsiveness between adjacent points, no less than $20^{\circ}$ apart, in every possible direction. Values of gradients were given as the percentage of response variation divided by the distance in degrees between the 2 points taken into account. The mean of the 3 best local gradients was then chosen as the mean value of gaze effect for that neuron. As indicated by Figures 5 and 6 , this value was variable among the neurons. The mean value was $2.2 \pm 0.7 \%$ (SD) of change in response per degree of gaze deviation. This value was significantly greater $(p<0.001)$ than that obtained from the group of neurons classified as non-gaze-dependent neurons $(0.9 \pm 0.3$; $n=80$ ), thus confirming that the 2 neuronal populations were different.

An attempt was also made to analyze which direction of gaze evoked the best visual response. Figure 7 shows the fixation locations at which V3A gaze-dependent cells were best activated by the receptive field stimulation. Figure 7 emphasizes 2 main points of our results. The first is that for the majority (51/77) of gaze-dependent neurons the firing rate in response to receptive field stimulation increased when the animal looked toward regions of the screen contralateral with respect to the hemisphere to which the neuron belonged. In particular, most of them increased their visual response when the animal looked toward the inferior contralateral quadrant, i.e., the same quadrant represented at the cortical level where recordings were made. The second point is that a preference for upward or downward directions, as well as for the straight-ahead direction, was never observed.

\section{Control experiments}

The above-described effect of gaze deviations upon the responsiveness of V3A neurons could have been due to inequalities in stimulus intensity between different sites on the screen. However, the screen area where stimuli were more effective differed from cell to cell, even in the same recording track (see Fig. 5), so we believe that inequalities in stimulus intensity were not responsible for the gaze effect.

\section{Control for background changes}

The observed gaze effect might be due to changes in the visual background as a consequence of the shifts of gaze toward different screen positions. However, we discount this possibility, since we used a screen that was uniformly illuminated and very large $\left(80^{\circ} \times 80^{\circ}\right)$ with respect to the receptive field sizes. If a suppressive or facilitatory zone outside the receptive field were responsible for the observed change in responsiveness, it would have had to be very large, well beyond what would be expected for a surround mechanism acting outside the receptive field. In a recent review on this subject, Allman et al. (1985) have reported that the maximal extent of such a surround would approximate 10 times the receptive field size. The neuron reported in Figure 4, for example, having a receptive-field size of about $2^{\circ}$, might have a surrounding zone with a diameter up to a maximum of $20^{\circ}$, that is, $10^{\circ}$ all around the receptive field, too small to interact with the screen borders, even when the animal lookcd at the most pcripheral screen locations (see Fig. 1).

Apart from the above considerations, several V3A neurons have been specifically tested to see if a change in visual background might explain the change in responsiveness observed with gaze deviations. Figure 8 shows the responses to visual stimulations of a gaze-dependent neuron firing maximally when the animal looked leftward, $15^{\circ}$ from the screen border. In that position, the receptive field was about $5^{\circ}$ from the screen border, so a facilitatory mechanism outside the receptive field might have been responsible for the increase in cell excitability. To test this possibility, the fixation point was moved to more rightward screen positions and the left part of the screen was covered by black cardboard in order to simulate the screen border and maintain the visual stimulation constant on that side of the receptive field. Despite this, the visual response progressively decreased from left to right, suggesting that the responsiveness of the cell really depended on the direction of gaze.

In a number of V3A neurons $(n=35)$, the background effect was tested by placing prisms in front of the animal's eyes in order to change the angle of gaze without changing the visual background, as previously done by Andersen et al. (1985) for gaze-dependent parietal neurons. The result was that both gazeand non-gaze-dependent neurons retained their characteristics even under these experimental conditions. Figure 9 shows an example in which a gaze-dependent neuron that was facilitated when the animal looked rightward was still strongly facilitated when the line of gaze was rightward displaced by a 20 diopter prism, although visual stimulation and actual position of the fixation point were unchanged at the center of the screen.

Finally, the responsiveness of 18 gaze-dependent neurons was tested in the standard 9 screen positions also in total darkness, 

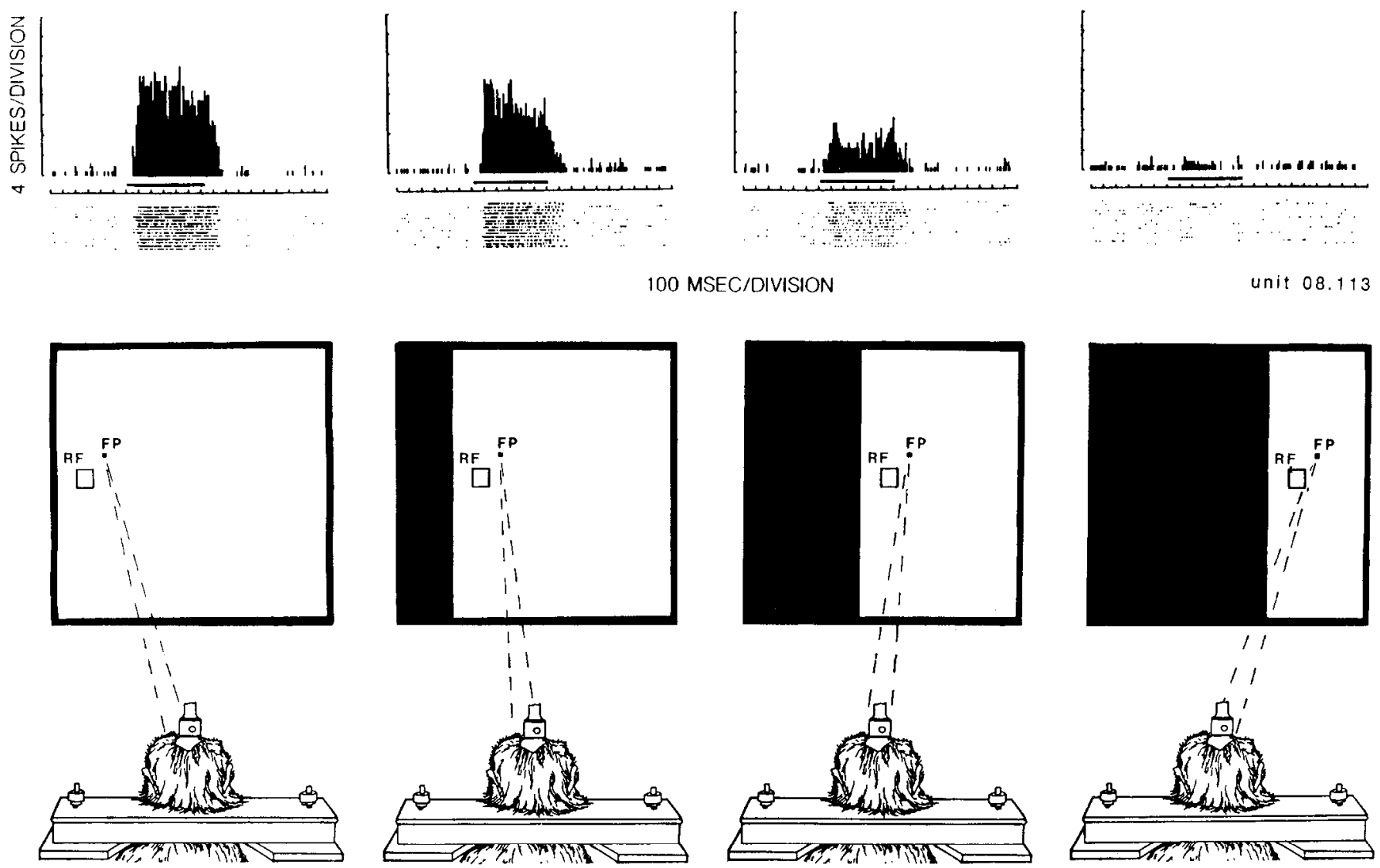

Figure 8. Influence of 4 different directions of gaze on the responsiveness of a V3A gaze-dependent neuron. Top, Peristimulus time histograms and replicas of impulse sequences showing the neuronal responses evoked by a $600 \mathrm{msec}$ stationary visual stimulus flashed on the receptive field while the animal looked at each of the 4 different screen positions. Fourteen trials for each screen position were carried out. Middle and bottom, Experimental arrangement. The large squares represent the screen; $F P$, fixation point; $R F$, receptive field. When the animal moved its eyes rightward from the leftmost screen position, the left part of the screen was covered by black cardboard in order to simulate a parallel displacement of the left screen border (the nearest to the receptive field) and therefore to maintain the visual stimulation outside the receptive field unchanged.

in order to completely eliminate any visual stimulation outside the receptive field. The results showed that, in darkness, the responsiveness of these neurons was still gaze-dependent, as shown in Figure 10.

\section{Control for depth changes}

The gaze effect upon cell responsiveness to visual stimulation might depend on differences in depth between fixation point and light stimulus (when the latter was on the receptive field), when they were presented in different positions on the screen. As a matter of fact, using a tangent screen, a stimulus on a receptive field located on the left of the fixation point, for example, is seen farther away than the fixation point when the animal looks to the left, and nearer than the fixation point when he looks to the right. In other words, gaze-dependent neurons might, in fact, be depth-sensitive neurons, and the gaze effect might simply be the result of changes in depth between stimulus and fixation point. This was certainly not the case for those gaze-dependent neurons whose receptive fields were on or near the vertical meridian (see Fig. 3) and which showed changes in responsiveness for horizontal gaze deviations because in such cases the difference in depth between the position of receptive field and that of the fixation point remained almost constant when the animal changed its gaze from left to right. The same was true for those neurons having receptive fields on or near the horizontal meridian and showing changes in responsiveness for vertical gaze deviations. In addition, it is worth noting that about $78 \%(69 / 88)$ of gaze-dependent neurons showed a gaze effect even on their spontaneous activity, i.e., without any intentional visual stimulation, hence without any depth cue (see, for example, the prestimulus activity in Fig. 10). Even in darkness, a gaze effect on spontaneous activity was still often present, as is also shown in the case of Figure 10.

\section{Receptive field characteristics and pattern of cortical distribution of gaze-dependent neurons}

Receptive fields of V3A neurons were mapped with stationary and moving stimuli variable in form, length, orientation, color, and direction of movement. We found a variety of neurons, some sensitive, others nonsensitive to each of these characteristics of the visual stimulus. There was a high incidence of orientation-selective cells: $55 \%$ of the total cell population $(n=$ 207) showed a range of responsive orientation smaller than $\pm 40^{\circ}$ from the preferred one. V3A neurons often responded with strong, sustained discharges of action potentials to stationary stimuli correctly oriented on their receptive fields, as well as to very slow stimulus movements. Most neurons were poorly sensitive to a single contrast border, as well as to very thin slits of light or dark bars, the most effective stimuli being of about the same size as the receptive field. Finally, several V3A neurons 

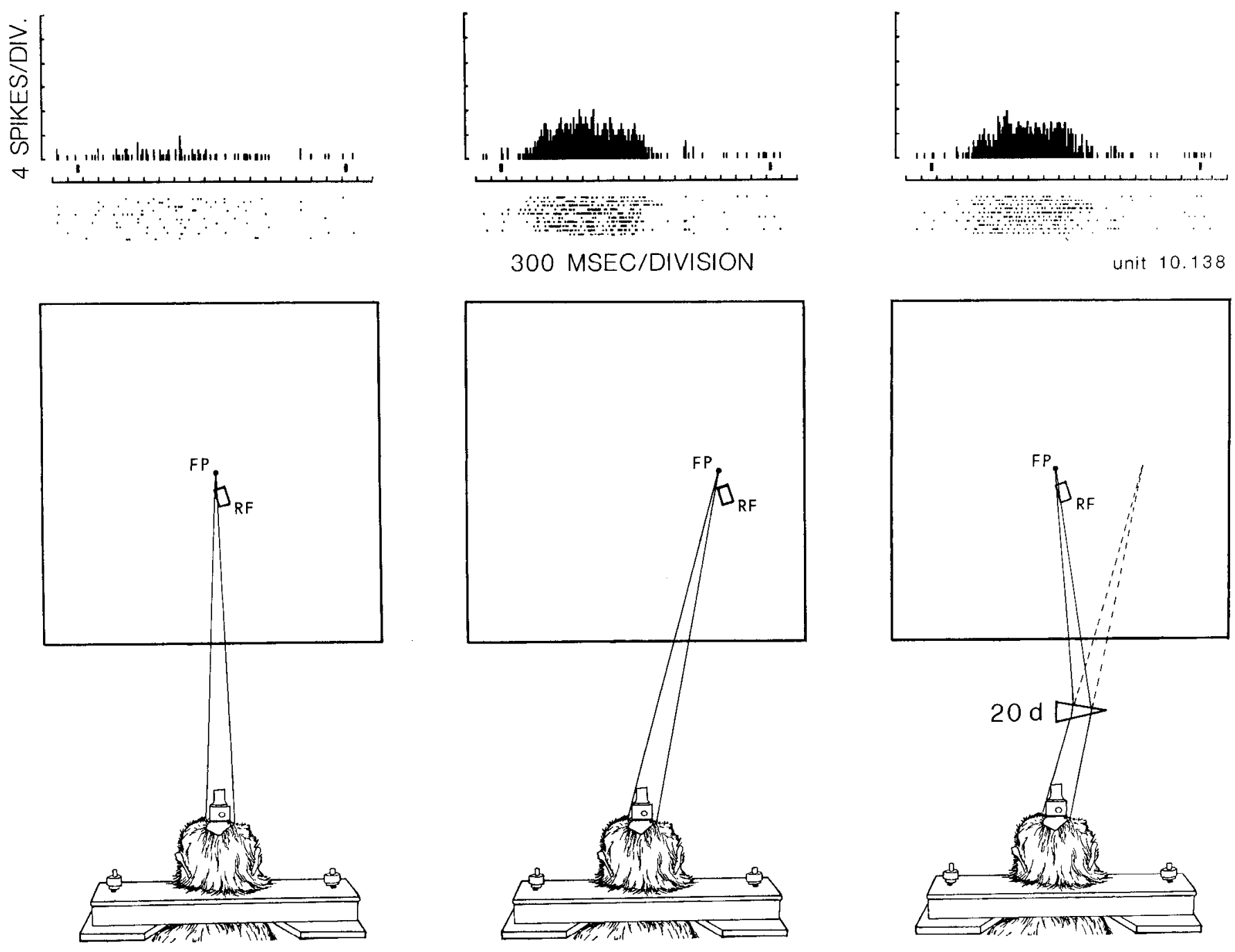

Figure 9. Gaze effect elicited by placing a prism in front of animal's eyes. Left, The animal looked at the center of the screen (FP, fixation point) while an optimal stimulus was moved across the receptive field $(R F)$ of a gaze-dependent neuron at a speed of about $1 \% / \mathrm{sec}$. Top left. Peristimulus time histograms and replicas of impulse sequences show that a very poor response was evoked by receptive field stimulation when the animal looked straight ahead. Middle, The fixation point (and, therefore, the receptive field) was displaced to the right and the neuron showed very good response to the same receptive field stimulation as on the left. Right, Fixation point location and receptive field stimulation were at the center of the screen, as on the left, but the direction of gaze was displaced by the same amount as in the middle by a 20 diopter prism placed along the line of gaze. Again, neuronal response was very strong, as in the middle.

showed a very strong spatial summation along the long axis of their receptive fields, whereas others were clearly end-stopped cells.

As to the gaze-dependent neurons of this prestriate area, no correlation at all was observed between the sensitivity to gaze direction and the sensitivity to either form, length, orientation, and direction of movement. No correlation was observed with the retinotopic location of the receptive field either: gaze-dependent and non-gaze-dependent neurons showed receptive fields located everywhere in the inferior contralateral quadrant, from the vertical to the horizontal meridian and from $1^{\circ}$ to $15^{\circ}$ from the fovea.

The population of gaze-dependent neurons studied is too small to permit an analysis of their laminar or columnar distribution inside area V3A. Nevertheless, our data suggest the existence of a segregation within this prestriate area. In most cases, all tested neurons recorded during single microelectrode penetrations were gaze-dependent (see filled circles on the center of
Figure 11) or non-gaze-dependent (see open circles). When both types of neurons were encountered along a single penetration (sce black-whitc circles), those of cach type were always clustered together, with no more than one change of type along the penetration. Figure 11 also shows that gaze-dependent neurons in area V3A were grouped together not only along single penetrations, but also over a discrete cortical region (see distribution of filled circles at the center of Fig. 11), and the same was true for the neurons not affected by gaze deviations (see open circle distribution). It seems reasonable to conclude that in the small part of V3A tested with close-spaced penetrations shown in Figure 11 (about $3 \times 3 \mathrm{~mm}$ ), there were at least 2 functionally distinct cortical regions, one full of gaze-dependent neurons and the other free of them. At present, we have not enough closespaced penetrations to reconstruct the complete pattern of distribution of gaze-dependent neurons in V3A. At any rate, we would point out that all the penetrations made in V3A showed the same pattern of distribution as described above. 


\section{LIGHT}
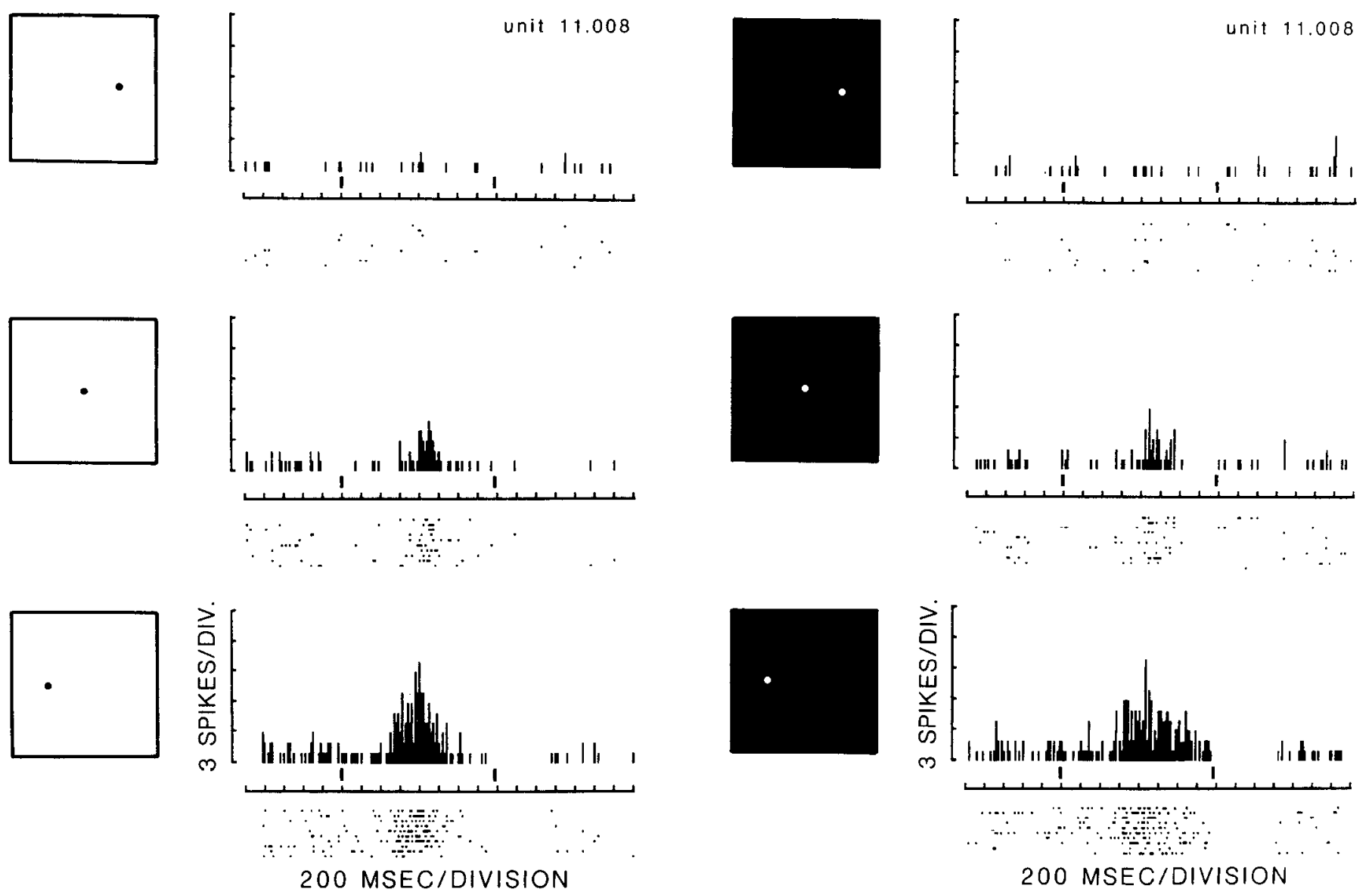

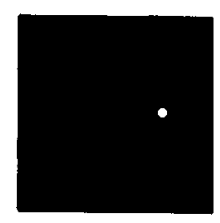

\section{DARKNESS}
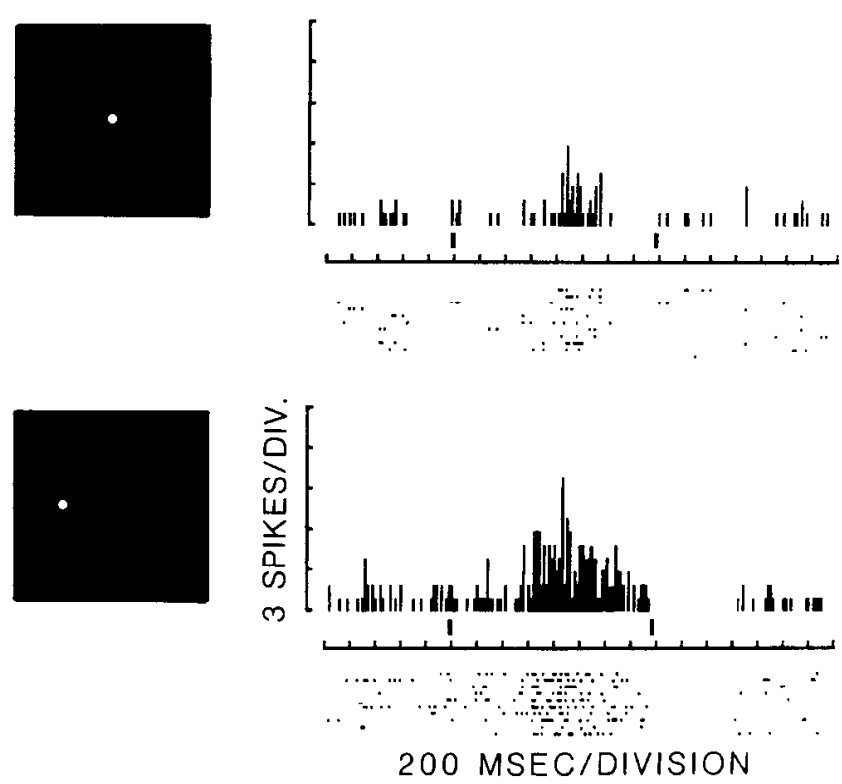

Figure 10. Pcrsistence of gaze effect in light and darkness. Left, The black spot inside each square represents the screen location of the fixation point. Only 3 different positions have been reported. Beside each of them, the neuronal responses to an optimal light stimulus that was moved across the receptive field (not shown) at a speed of about $5 \% \mathrm{sec}$ are reported as perstimulus time histograms and replicas of impulse sequences. Ten trials for each position were carried out. The visual background was a uniform white surface (the screen) with a luminance of about $2 \mathrm{~cd} / \mathrm{m}^{2}$. Right, The same neuron tested in darkness. In this series, light intensity of the visual stimulus was decreased by neutral density filters in order to achieve a brightness comparable to that used in the previous series (about 1 log unit above background level). Note that even the prestimulus activity of the cell was influenced by the direction of gaze both in light and darkness.

\section{Discussion}

In about half of V3A neurons, physically identical visual stimuli delivered to identical retinotopic positions (on their receptive fields) evoked different responses depending upon the direction of gaze. The influence of the direction of gaze upon cell responsiveness was generally not restricted to a particular region of the field of view. Rather, we observed a planar distribution of gaze effect on responsiveness, often spreading all over the tested gaze field (usually $40^{\circ} \times 40^{\circ}$ ) with different strengths for different gaze directions.

Control experiments were carried out to prevent inputs other than eye position in the orbit being responsible for the observed change in cell excitability. We discount the possibility that a sensory signal due to visual stimulation outside the receptive field might have been responsible for excitability change since this phenomenon was still present in a number of experimental situations in which visual stimulations outside the receptive field remained constant (by simulating displacements of the screen borders or by putting prisms in front of the animal's eyes). In addition, we observed that the gaze effect was often still present in total darkness, when no visual stimulation was acting outside the receptive field.

Our control experiments also discount the possibility that the observed phenomenon might have been due to differences in depth between the light stimulus on the receptive field and the fixation point, depending on the screen position the animal was looking at. We do not exclude that V3A neurons might be sensitive to depth; our results simply show that the gaze effect was still present both when the depth cue between fixation point and light stimulus remained constant (neurons with receptive field overlapping the vertical meridian) and when no depth cue at all was present (spontaneous activity of the cell, even in total darkness).

An alternative explanation of the results is that the phenomenon of change in responsiveness to visual stimulation was due to changes in fixation distance between different screen positions, the straight-ahead position being the nearest one for the animal. If this were the case, we would also have observed neurons with maximal or minimal responsiveness when the 


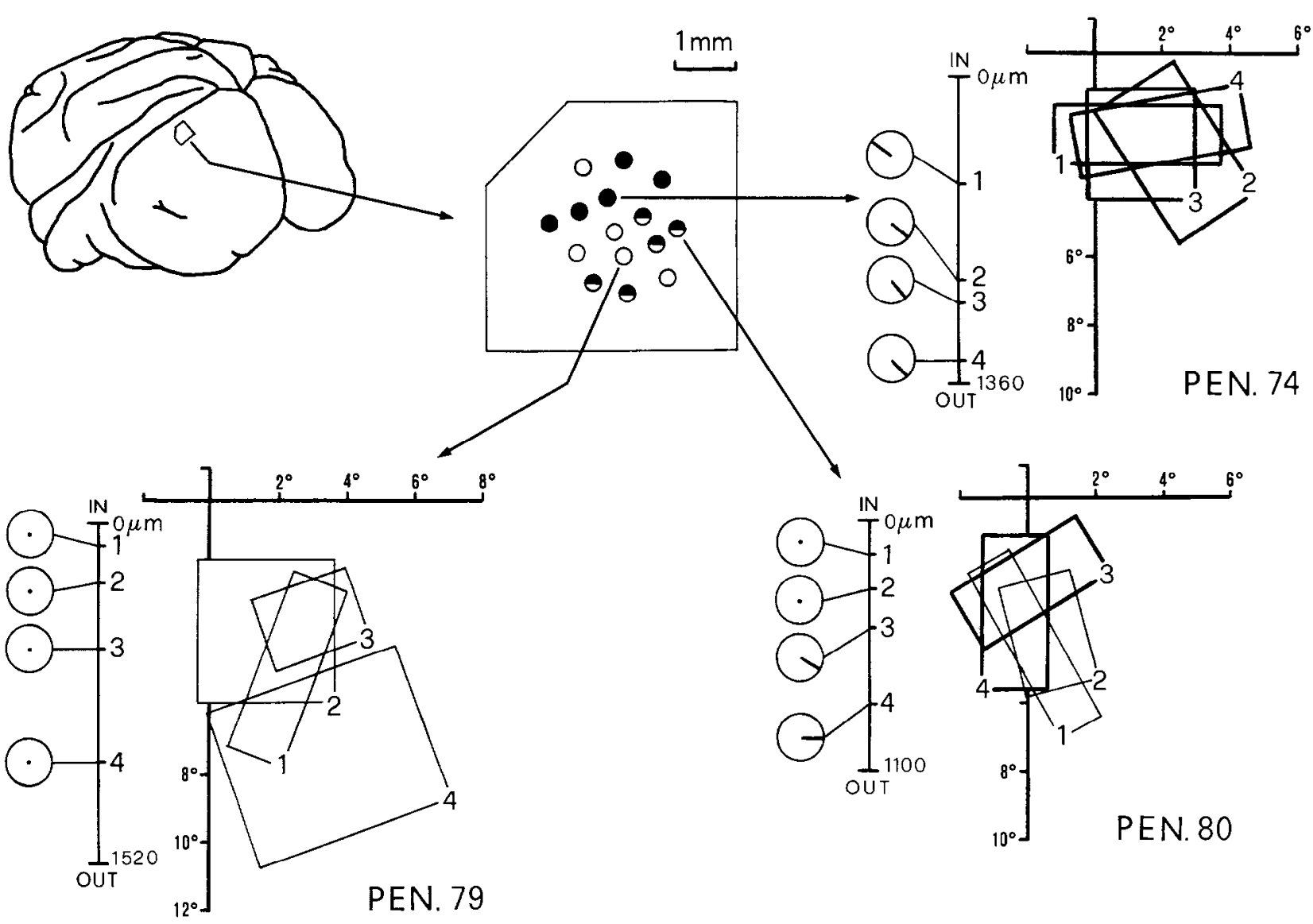

Figure 11. Pattern of cortical distribution of gaze-dependent neurons in a restricted region of area V3A. Top left, The region where 15 closespaced penetrations entered V1 to reach V3A in depth is shown. This region (a square with a cut angle) is shown enlarged at the center of the figure, where entry points of the penetrations are shown. Filled circles represent penetrations along which all neurons encountered were gazedependent in nature. Open circles indicate penetrations showing only non-gaze-dependent neurons, and black-white circles those where both types of neurons were encountered. Three penetrations are described in detail at the right and at the bottom. For each, receptive field location and size of the encountered neurons are shown (thick and thin lines have been used for gaze- and non-gaze-dependent neurons, respectively). Units marked $I$ and 2 in penetration (PEN.) 74 are the units 10.123 and 10.124 in Figure 5. To the left of each receptive field plot, a bar indicates the length (in $\mu \mathrm{m})$ of the penetration through area V3A $(I N-O U T)$ and the depths of the studied neurons with respect to the entry level in V3A. Circles beside units along the penetrations contain information about the gaze-dependent behavior of each neuron. The orientation of the line inside each circle indicates the mean direction of the increase in responsiveness with gaze deviations (see text and Fig. 7). In the case of non-gaze-dependent neurons, only a $d o t$ is shown at the center of the circle, indicating that the neuron was equally responsive to visual stimulations at any direction of gaze.

animal looked in the straight-ahead position (which was the nearest one) and the reverse behavior in all the remaining screen positions, all of them being farther than the straight-ahead one. On the contrary, we never observed such a gaze-related distribution of responsiveness in any of the tested V3A neurons.

Andersen and Mountcastle (1983) showed that the gaze effect on responsiveness to visual stimulations of inferior parictal lightsensitive neurons depended upon the behavioral state of the animal. Unfortunately, we were unable to repeat their experimental schedule in order to test the effect of directed visual attention on cell excitability. The small receptive field size of V3A neurons with, sometimes, inhibitory or antagonistic regions and the long time required to activate them when a slowmoving stimulus was the most effective one, made it very difficult to activate them correctly while the animal was looking around during relaxed wakefulness. Although we have no information about the influence of behavioral state on cell excitability in V3A, we discount that what we consider to be a gazedependent effect might be due to changes in the behavioral state of the animal. In fact, the animal had to pay constant visual attention during the experimental task and the different screen positions were chosen in a random order and sometimes repeated.

We conclude that the direction of gaze is really an input modulating the excitability of part of the V3A neurons, though we are also aware that other events, like changes in retinal disparity and dirceted visual attention, might modulate ncuronal cxcitability in this prestriate area of the visual cortex.

\section{Neural mechanism of gaze effect}

At present, we have no direct experimental evidence concerning the neural mechanism that allows the eye position to modulate responsiveness to visual stimulation of V3A neurons. One possibility is that gaze-dependent visual neurons of area V3A receive an input from the same type of neurons of other structures of the visual system. So far, thalamic intralaminar nuclei of the cat and posterior parietal cortex of the monkey have been shown to contain neurons whose responsiveness to visual stimulation of their receptive field is modulated by the eye position (Schlag et al., 1980; Andersen and Mountcastle, 1983); however, in both 
cascs the organization and size of the receptive fields are very different from those observed in area V3A. Pulvinar nuclei are known to receive inputs from several structures connected with eye movements besides receiving visual inputs and project massively to the extrastriate visual cortex but do not seem to contain gaze-dependent visual neurons (Petersen et al., 1985). Other structures of the visual system, possibly connected with area V3A, have still not been studied for this.

A second possibility is that the eye-position signal directly modulates the excitability of V3A gaze-dependent neurons. In this regard, among the structures that might be directly connected with area V3A, the frontal eye field (area 8), the posterior parietal cortex (area 7a) and the intralaminar nuclei of the thalamus all contain neurons whose spontaneous firing rate in darkness is a function of the eye position in the orbit (Bizzi, 1968; Sakata et al., 1980; Schlag-Rey and Schlag, 1984), but their conncction with area V3A has not yet been demonstrated.

Among these structures, intralaminar nuclei seem to be a likely source of eye position input for gaze-dependent neurons since electrical stimulation of those nuclei enhances cortical visual responses (Hunsperger and Roman, 1976), and, also, they represent a nodal point linking several cortical and subcortical structures, all involved in the control of gaze (see Schlag and Schlag-Rey, 1986, for references on this subject). In addition, eye-position cells in the intralaminar nuclei have several properties similar to those of the gaze-dependent visual neurons described in this paper (Schlag-Rey and Schlag, 1984). Among these properties are: the range of eye position modulating their firing rate, and the fact that eye-position units located in the rostral part of the nucleus lateralis dorsalis increased their firing rate when the animal looked contralaterally with respect to the site of recording, while none of them increased its frequency of discharge by looking upward or downward.

\section{Comparison with posterior parietal gaze-dependent neurons}

The receptive fields of $\mathrm{V} 3 \mathrm{~A}$ gaze-dependent neurons are small and, in most cases, optimally activated by visual stimuli of about the same size of the receptive field. On the contrary, gaze-sensitive visual neurons of area $7 \mathrm{a}$ are very large, often covering over $80^{\circ}$ of visual field, and well activatcd by visual stimuli much smaller than the receptive field size (Andersen and Mountcastle, 1983; Andersen et al., 1985; Zipser and Andersen, 1988). The functional relevance of this difference is discussed next.

Apart from receptive field size, 3 other major differences are observed when the responses of $\mathrm{V} 3 \mathrm{~A}$ gaze-dependent neurons are compared with those observed in the inferior parietal lobule. The first is that many V3A gaze-dependent neurons were contralaterally facilitated, whereas in area 7a no laterality effect was observed (Andersen and Mountcastle, 1983). In this behavior, there is an analogy with the receptive field location in the 2 areas: in area $\mathrm{V} 3 \mathrm{~A}$, receptive fields are always contralaterally located; in area 7a, they frequently occupy both ipsi- and contralateral sides of the visual field (Motter and Mountcastle, 1981). The second difference is that we did not find neurons best- or least-facilitated in the straight-ahead direction, whereas in area $7 \mathrm{a}$ about half of gaze-dependent neurons showed either maximal or minimal responsiveness to visual stimulation for fixation in straight-ahead direction (Andersen and Mountcastle, 1983). The third difference is that we never observed a preference for upward or downward directions, while in area $7 \mathrm{a}$ both have been described (Andersen and Mountcastle, 1983). We, too, found changes in responsiveness in the vertical directions, but the horizontal changes were always stronger than the vertical ones or, at most, the same; therefore, the preferred direction never was a purely upward or downward.

As far as the gradient of change in responsiveness to visual stimulation is concerned, we found a mean value of $2.2 \pm 0.7 \%$ change in the response per degree of gaze deviation in area $\mathrm{V} 3 \mathrm{~A}$, while Andersen and Mountcastle (1983) reported a value of 3.65 for gaze-dependent neurons in area 7a. Ilowever, we used a mean value (the mean of the 3 best) to quantify the gradient of each neuron, while Andersen and Mountcastle used the value obtained by the steepest part of the response/angle gaze function. Therefore, the disparity in the gradients between areas V3A and 7 a may be due to methodological differences rather than actual changes in responsiveness.

Finally, it has been reported that parietal gaze-dependent neurons were about $60 \%$ of the neuronal population (Andersen and Mountcastle, 1983; Andersen et al., 1985; Zipser and Andersen, 1988), while in area V3A they represent almost $50 \%$ of our sample. The difference can hardly be considered significant, given the relatively small neuronal samples in the 2 cortical areas and the clustered distribution of gaze-dependent neurons observed in area $V 3 \mathrm{~A}$.

\section{Functional role of V3A gaze-dependent visual neurons}

As pointed out in our introductory remarks, McKay (1973) postulated that the stability of visual perception despite eye and/ or body movements would be due to the existence of an objective map of the visual world. According to the "evaluating hypothesis" of MacKay, the neuronal network subserving the objective map would continuously "evaluate" whether something had changed its spatial location (real position) in the visual environment. A direct neuronal correlate of this objective map might be the existence of "real-position" detectors, that is, visual neurons with very large receptive fields responsive to stimuli occupying a particular region of visual space, regardless of the position of the eye in the orbit and, consequently, of the retinal locus actually activated (see Sparks and Mays, 1983). To date, cells of this type have not yet been identified in the visual system.

Gaze-dependent neurons of area V3A have visual receptive fields that arc small in size and remain strictly retinotopic; in addition, the magnitude of their response to visual stimulation is clearly modulated by the eye position in the orbit. Because of the smallness of the receptive fields, eye displacements of more than a few degrees take them on completely different locations of the field of view. Thus, when the animal changes the direction of gaze, a single receptive field receives visual information from different spatial locations, and the same receptive field stimulation evokes different firing rates, according to the eye position in the orbit. This means that the eye movements made to explore the visual environment would allow a gazedependent neuron to directly encode the objective locations of a large part of the field of view into different firing rates, as shown in Figure 5.

The encoding process of a single gaze-dependent neuron, although broad in extent thanks to the eye movements, is not very precise because different objective locations are coded with the same discharge frequency (see isoresponsiveness lines in Fig. 6). However, if several V3A neurons with different distributions of responsiveness were taken into account, an improvement in the spatial resolution of the encoding process would result. Present data show that gaze-dependent neurons are widely represented in area V3A, and those with different distribution of 
responsiveness are often grouped together. Therefore, it may be suggested that these neurons, together, form a neuronal network allowing a precise encoding process of visual space in a head frame of reference. The same suggestion has already been advanced for gaze-dependent visual neurons of area $7 \mathrm{a}$ (Andersen et al., 1985; Zipser and Andersen, 1988), but some differences appear evident between these neurons and those of area V3A. Gaze-sensitive visual neurons of area $7 \mathrm{a}$ have very large receptive fields (often over $80^{\circ}$ in diameter) with complex profiles of excitability (see Zipser and Andersen, 1988). On the one hand, this means that neurons of this type would be able to encode a large part of the visual field even when the animal maintains the same direction of gaze, while this is not the case for V3A neurons. On the other hand, when the animal changes the direction of gaze, a visual stimulus in the same spatial location might evoke different frequencies of discharge by the same neuron, since it falls on different parts of the receptive field. Again, this is not the case for the gaze-dependent neurons of area V3A. But it does not seem to be the case for area 7 a either. As a matter of fact, Andersen et al. (1985) demonstrated that in area 7a the interaction between the gaussian profile of excitability of the receptive field and the quite linear gaze effect of visual responsiveness might allow an encoding process of visual space in head-centered coordinates. We believe that area V3A performs a spatial transformation of the retinotopical organized visual information, perhaps with a different strategy with respect to area $7 \mathrm{a}$, as V3A neurons have small receptive fields with quite a uniform profile of excitability. We do not claim that the construction of an objective map of the field of view is the only function of area V3A, firstly because only about half of its neurons were gaze-dependent in nature and, secondly, because several other characteristics of the visual stimulus were found to activate V3A neurons selectively, whether or not they were gazeor non-gaze-dependent. However, it seems reasonable to believe that the construction of an objective map of the visual field is at least one function of area V3A.

The question now arises as to why, if the functional role suggested for gaze-dependent visual neurons is correct, objective spatial maps are present in several structures of the visual system (area $7 \mathrm{a}$, area V3A, internal medullary lamina of the thalamus, and, perhaps, many others still not studied for this characteristic). It might be suggested that each of these structures uses its own representation of the visual space for its own needs, depending on the role the structure plays in the visual process. For example, area $7 \mathrm{a}$, which is known to be concerned with processing of spatial information and perception of spatial relationships, would need an objective map of the visual environment for visuomotor coordination in spatially oriented behavior (see Andersen and Mountcastle, 1983) and for motor actions directed to locations in space (see Andersen et al., 1985). It has been suggested that also the superior colliculus would need an objective map to locate saccade targets in a spatial, rather than in a retinocentric, frame of reference (see Mays and Sparks, 1980; Sparks and Mays, 1983). We believe that each of the extrastriate cortical visual areas which, like area $\mathrm{V} 3 \mathrm{~A}$, is probably concerned with specific and different visual processes such as analysis of form, color, or movement (Zeki, 1985), needs an objective map of the field of view just like V3A to allow perceptual visual stability despite eye movements.

Regarding the organization of this map, our results show that gaze-dependent neurons are grouped together, within area V3A, and are probably present in all cortical layers, but they seem tangentially segregated in restricted cortical regions (see Fig. 11). This pattern of distribution might reflect a modular organization, like that described in areas V1 and V2, where "blobs" and "stripes" have been shown to contain different functional units (Livingstone and Hubel, 1984; Shipp and Zeki, 1985). In any case, further experiments with a larger number of close-spaced penetrations are needed to confirm this suggestion and to build a complete picture of the cortical distribution and organization of gaze-dependent neurons within area V3A.

\section{References}

Aicardi, G., P. P. Battaglini, and C. Galletti (1987) The angle of gaze influences the response to visual stimulation of cells in V3-complex of macaque monkcy visual cortex. J. Physiol. (Lond.) 390:27P.

Allman, J., F. Miezin, and E. McGuiness (1985) Stimulus specific responses from beyond the classical receptive field: Neurophysiological mechanisms for local-global comparisons in visual neurons. Annu. Rev. Neurosci. 8: 407-430.

Andersen, R. A., and V. B. Mountcastle (1983) The influence of the angle of gaze upon the excitability of the light-sensitive neurons of the posterior parietal cortex. J. Neurosci. 3: 532-548.

Andersen, R. A., G. K. Essick, and R. M. Siegel (1985) Encoding of spatial location by posterior parietal neurons. Science $230: 456-458$.

Battaglini, P. P., S. Squatrito, C. Morandi, and C. Galletti (1984) Microprocessor-based system for spike and eye movement acquisition and storage. Pfluegers Arch. 400: 194-196.

Battaglini, P. P., C. Galletti, and G. Aicardi (1987) The activity of cells in the visual cortex of the annectant gyrus may be influenced by the angle of gaze. Pfluegers Arch. 408: S39.

Bizzi, E. (1968) Discharge of frontal eye field neurons during saccadic and following eye movements in unanesthetized monkeys. Exp. Brain Res. 6: 69-80.

Fuchs, A. F., and D. A. Robinson (1966) A method for measuring horizontal and vertical eye movement chronically in the monkey. J. Appl. Physiol. 21: 1068-1070.

Galletti, C., S. Squatrito, P. P. Battaglini, and M. G. Maioli (1984) "Real motion" cells in the primary visual cortex of macaque monkeys. Brain Res. 301: 95-110.

Galletti, C., P. P. Battaglini, and G. Aicardi (1988) "Real-motion" cells in visual area V2 of behaving macaque monkeys. Exp. Brain Res. 69: 279-288.

Hunsperger, R. W., and D. Roman (1976) The integrative role of the intralaminar system of the thalamus in visual orientation and perception in the cat. Exp. Brain Res. 25: 231-246.

Judge, S. J., B. J. Richmond, and F. C Chu (1980) Implantation of magnetic search coils for measurement of eye positions: An improved method. Vision Res. 20:535-538.

Livingston, M. S., and D. H. Hubel (1984) Anatomy and physiology of a color system in the primate visual cortex. J. Neurosci. 4: 309356.

MacKay, D. M. (1973) Visual stability and voluntary eye movements. In Handbook of Sensory Physiology, Vol. VII/3, Central Processing of Visual Information. Part A: Integrative Functions and Comparative Data, R. Jung, ed., pp. 307-332, Springer, New York.

Mays, L. E., and D. L. Sparks (1980) Dissociation of visual and saccade-related responses in superior colliculus neurons. J. Neurophysiol. 43: 207-232.

Motter, B. C., and V. B. Mountcastle (1981) The functional properties of the light-sensitive neurons of the posterior parietal cortex studied in waking monkeys: Foveal sparing and opponent vector organization. J. Neurosci. 1: 3-26.

Petersen, S. E., D. L. Robinson, and W. Keys (1985) Pulvinar nuclei of the behaving rhesus monkey: Visual responses and their modulation. J. Neurophysiol. 54: 867-886.

Sakata, H., H. Shibutani, and K. Kawano (1980) Spatial properties of visual fixation neurons in posterior parietal association cortex of the monkey. J. Neurophysiol. 43: 1654-1672.

Schlag, J., and M. Schlag-Rey (1986) Role of the central thalamus in gaze control. In The Oculomotor and Skeletalmotor Systems: Differences and Similarities, H. J. Freund, U. Buttner, B. Cohen, and J. Noth, eds., Prog. Brain Res. 64: 191-201, Elsevier, New York.

Schlag, J., M. Schlag-Rey, C. K. Peck, and J. P. Joseph (1980) Visual 
responses of thalamic neurons depending on the direction of gaze and the position of the target in space. Exp. Brain Res. 40: 170-184.

Schlag-Rey, M., and J. Schlag (1984) Visuomotor functions of central thalamus in monkey. I. Unit activity related to spontaneous eye movements. J. Neurophysiol. 51: 1149-1174.

Shipp, S., and S. Zeki (1985) Segregation of pathways leading from area V 2 to areas V4 and V 5 of macaque monkey visual cortex. Nature 315: 322-325.

Sparks, D. L., and L. E. Mays (1983) Role of the monkey superior colliculus in the spatial localization of saccade targets. In Spatially Oriented Behavior, A. Hein and M. Jeannerod, eds., pp. 63-85, Springer, New York.

Suzuki, H., and M. Azuma (1976) A glass-insulated "elgiloy" microelectrode for recording unit activity in chronic monkey experiments. Electroencephalogr. Clin. Neurophysiol. 41: 93-95.

Tomlison, R. D., and P. S. Bahra (1986) Combined eye-head gaze shifts in the primate. I. Metric. J. Neurophysiol. 56: 1542-1557.
Van Essen, D. C., and S. M. Zeki (1978) The topographic organization of rhesus monkey prestriate cortex. J. Physiol. (Lond.) 277: 193-226.

Zeki, S. M. (1978) The third visual complex of rhesus monkey prestriate cortex. J. Physiol. (Lond.) 277: 245-272.

Zeki, S. (1985) The mapping of visual functions in the cerebral cortex. In Brain Mechanisms of Sensation, Y. Katsuki, R. Norgren and M. Sato, eds., pp. 105-128, Wiley, New York.

Zeki, S. M., and D. R. Sandeman (1976) Combined anatomical and electrophysiological studies on the boundary between the second and third visual areas of rhesus monkey cortex. Proc. R. Soc. London [Biol.] 194: 555-562.

Zipser, D. and R. A. Andersen (1988) A back-propagation programmed network that simulates response properties of a subset of posterior parietal neurons. Nature 331: 679-684. 